ИЗВЕСТИЯ АКАДЕМИИ НАУК ЭСТОНСКОИ ССР. ТОМ 27 ФИЗИКА * МАТЕМАТИКА. 1978, № 4

\title{
SYMMETRIES ARISING IN GENERALIZED STRING ACTION
}

\section{A. AINSAAR, K. KIIRANEN, M. KOIV. OLdISTATUd STRINGI MOJUST TUlENEVAD SOM- MEETRIAD}

А. АИНСААР, К. КИИРАНЕН, М. КЫИВ. СИММЕТРИИ, ВОЗНИКАЮЩИЕ ПРИ ОБОБЩЕНИИ ДЕНСтвия СтРунЫ

\author{
(Presented by P. Kard)
}

The action integral of a relativistic string is generalized to $n$-dimensional objects in $m$-dimensional space. It can be brought to the action for $m-n$-component fields in $n$ dimensional space. We call such object «sympleon». The corresponding equation is hodograph invariant via the equivalence of the initial coordinates. In the case of one-component field the scalar Born-Infeld equation is obtained.

The action integral for a relativistic string, which is closely related to dual models $\left[{ }^{1,2}\right]$, is known to be

$$
S=-\alpha \iint d \xi^{0} d \xi^{1} \sqrt{\left(\frac{\partial x^{\mu}}{\partial \xi^{0}} \frac{\partial x_{\mu}}{\partial \xi^{1}}\right)^{2}-\left(\frac{\partial x^{\mu}}{\partial \xi^{0}}\right)^{2}\left(\frac{\partial x^{\nu}}{\partial \xi^{1}}\right)^{2}},
$$

where $\xi^{0}$ and $\xi^{1}$ are the internal parameters of the string, $\xi^{0}$ being proper time and $\xi^{1}$ any longitudinal parameter. This action is obtained by means of a generalization of the action integral for relativistic point mass where only one parameter $\xi^{0}$ is involved.

Such a generalization can be continued. For instance, when taking 3 parameters $\xi^{0}, \xi^{1}, \xi^{2}$, a corresponding relativistic membrane is obtained.

The action integral for an $n$-dimensional object moving in $m$ dimensional space $(m>n)$ is

$$
S=-\alpha \iint \ldots \int d \xi^{0} d \xi^{1} \ldots d \xi^{n} \sqrt{-\operatorname{det} g},
$$

where

$$
g_{i k}(\xi)=-\frac{\partial x^{\lambda}}{\partial \xi^{i}} \frac{\partial x_{\lambda}}{\partial \xi^{k}} \equiv-x_{, i}^{\lambda} x_{\lambda, k}, \quad(i, k=0,1, \ldots, n,
$$

The coordinates $x^{\lambda}$ of the object are functions of the parameters $\xi^{i}$ :

$$
x^{\lambda}=x^{\lambda}\left(\xi^{0}, \xi^{1}, \ldots, \xi^{n}\right) .
$$

Now, one can consider $n+1$ coordinates, say $x^{0}, x^{1}, \ldots, x^{n}$, as independent and the remaining $m-n$ ones as their functions

$$
x^{\alpha} \equiv \varphi^{\alpha}\left(x^{0}, x^{1}, \ldots, x^{n}\right), \quad(\alpha=n+1, \ldots, m) .
$$

The quantities $\varphi^{\alpha}$ resemble certain $m-n$-component fields in $n$ dimensional space.

Next, a question of interest is what Lagrangian and field equation follow for these fields.

The matrix $g$ can be split into two parts: 


$$
g_{i k}=-\left(x_{, i}^{\mu} x_{\mu, k}+\varphi_{, i}^{\alpha} \varphi_{\alpha, k}\right), \quad \begin{aligned}
& \mu=0,1, \ldots, n, \\
& \alpha=n-1, \ldots, m) .
\end{aligned}
$$

Since $\varphi^{\alpha}$ are functions of $x^{\mu}$, their derivatives can be expressed as

$$
\varphi_{\varphi, i}^{\alpha}=\frac{\partial \varphi^{\alpha}}{\partial x^{\mu}} \frac{\partial x^{\mu}}{\partial \xi^{i}} \equiv \varphi_{, \mu}^{\alpha} x_{, i}^{\mu}
$$

and (6) takes the form

where

$$
g_{i k}=-x_{, i}^{v} \Phi_{v}^{\mu} x_{\mu, k}
$$

$$
\Phi_{v}^{\mu}=\delta_{v}^{\mu}+\varphi_{, v}^{\alpha} \varphi_{\alpha}^{, \mu} .
$$

Following the rule for the determinant of a matrix product, one can obtain from (8)

$$
\sqrt{-\operatorname{det} g}=\frac{1}{G} \sqrt{\operatorname{det} \Phi}
$$

where $\frac{1}{G}$ equals the transition Jacobian between the two sets of coordinates $\xi$ and $x$

$$
\frac{1}{G}=\operatorname{det} x_{, i} .
$$

Thus, action (2) takes the form

$$
S=-\alpha \iint \ldots \int d x^{0} d x^{1} \ldots d x^{n} \sqrt{\operatorname{det} \Phi} .
$$

The integration variables could be changed via remarkable property (10) which enables factorizing the Lagrangian to a corresponding Jacobian. We call an object with such property «sympleon» (we keep in mind two Greek words meaning «quite full» and «that floating together»).

If $m=n+1$, the field $\varphi^{\alpha}$ has only one component and it is easy to get the Lagrangian

$$
L=-\alpha \sqrt{1-\varphi, \mu \varphi^{\mu}}, \quad(\mu=0,1, \ldots, n)
$$

which belongs to the scalar Born-Infeld equation. For $n=1$ the equation is

$$
\left(1-\varphi_{, 0}^{2}\right) \varphi, 11+2 \varphi, 1 \varphi, 0 \varphi, 01-\left(1+\varphi_{, 1}^{2}\right) \varphi, 00=0 .
$$

A relation between (1) and (14) was shown in $\left[{ }^{2}\right]$ and it is of an essential significance, because the scalar Born-Infeld equation (14) has soliton solutions. So there exists a certain connection between the two equations belonging to different extended-particle models - the string and soliton ones.

Another significant feature of equation (14) is that it is hodograph invariant, i.e., it conserves its shape when interchanging the roles of the field function and the coordinates. The hodograph invariance is a consequence of the fact that this equation is obtained from sympleon action integral (2), where arbitrary coordinates can principally be taken as field components. The fact is that every field equation obtained in this way from sympleon action integral (2) is hodograph invariant.

For vector fields $(n=3)$ it should be mentioned that the equation obtained from (2) and (3) is not gauge invariant. This means that all the derivatives cannot be involved in the forms 


$$
F_{\mu v}=\varphi_{v, \mu}-\varphi_{\mu, v} .
$$

This is why we cannot get the Born equation Lagrangian $\left[{ }^{3}\right]$

$$
L \sim \sqrt{1-\frac{1}{2} F_{\mu \nu} F_{\mu \nu}},
$$

which is a three-dimensional generalization of (13).

Action (2) with (3) is not the only form for a sympleon. For instance, if the space-time dimension is even, there can be $2(n+1)=m+1$ and instead of (3) the symplectic scalar product can be applied in (2):

$$
\tilde{g}_{i k}=\left\langle x_{i}, x_{k}\right\rangle \equiv x_{, i}^{\mu} x_{, k}^{\mu+n+1}-x_{, k}^{\mu} x_{, i}^{\mu+n+1} .
$$

In case $n=3$ it leads, instead of $(8)$, to

$$
\tilde{g}_{i k}=F_{\mu v} x_{, i}^{\mu} x_{, h}^{\imath},
$$

which obviously describes a sympleon and, besides, leads to a gauge invariant Lagrangian. Since there is no field equation corresponding to it, one should take a linear combination instead:

$$
a g_{i k}+b \tilde{g}_{i k},
$$

but now the gauge invariance is lost again. The latter can be restored only by declining from form (19).

When taking instead of (3)

where

$$
g^{\prime}{ }_{i k}+i \widetilde{g}_{i k},
$$

$$
g^{\prime}{ }_{i k}=-x_{, i}^{\mu} x_{\mu, k}
$$

is the first term in (6), then the Born-Infeld vector equation with Lagrangian $\left[{ }^{4}\right]$

where

$$
L \sim \sqrt{1+F-G^{2}},
$$

$$
\begin{gathered}
F=\frac{1}{2} F_{\mu v} F^{\mu v}, \\
G=\frac{1}{8} \varepsilon_{\alpha \beta \mu v} F^{\alpha \beta} F^{\mu v}
\end{gathered}
$$

can be obtained. Here the hodograph invariance is lost via cutting down equation (6). Apparently, in the considered approach, these two symmetries are mutually exclusive.

The authors are indebted to A. Parring for helpful discussions.

\section{REFERENCES}

1. S cherk, J. An introduction to the theory of dual models and strings. - Rev. Mod. Phys., 1975 , v. $47, \mathrm{~N} 1$, p. $123-164$.

2. Бар башов Б. М., Ч ер н и ко в Н. А. Решение и квантование нелинейной двухмерной модели типа поля Борна-Инфсльда. - ЖЭТФ, 1966, т. 50, вып. 5, c. $1296-1308$.

3. Born, M. On the quantum theory of electromagnetic field. - Proc. Roy. Soc. A, 1934 , v. 143 , N 843 , p. $410-437$.

4. B or n, M., I n f e Id, L. Quantization of the new field theory. - Proc. Roy. Soc. A, 1934 , v. 147 , N 862 , p. $522-546$. 